\title{
Seyrek Genelleştirilmiş Ofset Polinom Eğrisinin Prony Algoritması ile Olușturulması
}

\author{
Ali Çalışkan ${ }^{1}$, Vahide Bulut ${ }^{2}$, Selcan Kocabaş ${ }^{3 *}$ \\ ${ }^{1}$ Kırklareli Üniversitesi, Fen Edebiyat Fakültesi, Matematik Bölümü, Kırklareli, Türkiye, (ORCID: 0000-0002-2693-3269), acalıskan@klu.edu.tr \\ 2 İzmir Katip Çelebi Üniversitesi, Mühendislik ve Mimarlık Fakültesi, Mühendislik Bilimleri Bölümü, İzmir, Türkiye (ORCID: 0000-0002-0786-8860), \\ vahide.bulut@ikcu.edu.tr \\ $3^{3 *}$ Kırklareli Üniversitesi Fen Edebiyat Fakültesi, Matematik Bölümü, Kırklareli, Türkiye (ORCID: 0000-0003-3416-9608), 1208251101@ogrklu.edu.tr
} (International Conference on Design, Research and Development (RDCONF) 2021 - 15-18 December 2021)

(DOI: 10.31590/ejosat.1041047)

ATIF/REFERENCE: Çalışkan, A., Bulut, V. \& Kocabaş, S. (2021). Seyrek Genelleştirilmiş Ofset Polinom Eğrisinin Prony Algoritması ile Oluşturulması. Avrupa Bilim ve Teknoloji Dergisi, (32), 553-556.

\section{$\ddot{O} \mathbf{z}$}

Bilgisayar donanımındaki teknolojik gelişmeler, bilim adamlarının bilimsel hesaplama ile ele aldığı sorunların boyutunu ve karmaşıklığını büyük ölçüde genişletmesine izin vermiştir. Seyrek polinomlar, hemen hemen her bilgisayar uygulamasında bulunmakta olup sıfır katsayılı polinomlar pratik ortamlarda sıklıkla ortaya çıkmaktadır. Son yıllarda, güç temelinden farklı terim temeli kullanılarak seyrek bir polinomun interpolasyonu için çeşitli algoritmalar tasarlanmıştır. Prony'nin 18. yüzyıldaki klasik sayısal tekniği Ben-Or ve Tiwari tarafından yeniden keşfedilerek yaklaşık 200 yıl sonra bilgisayar alanına uyarlanmıştır.

Pratik hayatta seyrek polinomların karşımıza çıktığı durumalara baktığımızda bunlar arasında görüntü netleştirme ve ses dalgalarının ayarlanması gibi problemler yer almaktadır. Fotoğraf işleme, onu gerçeğine yakın bir hale getirme son yıllarda büyük ilerlemeler kaydederken günümüzde orman, gün batımı, gökkuşağı gibi manzaraların dijital görüntüleri benzeri görülmemiş bir gerçekçilikle sentezlenebilmektedir. Bununla birlikte, kamera merceği ve ses dalgaları ile ilgili fenomenleri simüle etmek hala zorlu bir problem olmaya devam etmektedir.

Yapılacak olan çalışmada, merceklerin odak bulanıklığı, sapmalarının ve ses dalgalarının ayarlanmasında kullanılan seyrek polinomlara geometrik bir perspektiften yaklaşılarak seyrek genelleştirilmiş ofset eğrileri bulunacaktır. Bu seyrek genelleştirilmiş ofset polinom eğrisi, Prony algoritması ile eğrinin değerleri kullanılarak yeniden elde edilecektir. Orijinal seyrek polinom ile seyrek genelleştirilimiş̧ ofset polinom eğrisi ile arasındaki ilişki incelenecektir. Böylece bu eğriler kullanılarak kamera mercek ve ses dalgalarının ince ayarlarının yapılmasına bilgisayar destekli geometrik tasarım ve hesaplama yöntemleriyle katkıda bulunulacaktır.

Anahtar Kelimeler: Seyrek Polinomlar, Prony Algoritması, Seyrek Genelleştirilmiş Ofset Polinom Eğrisi.

\section{Forming The Sparse Generalized Offset Polynomial Curve by Prony Algorithm}

\begin{abstract}
Technological advances in computer hardware have allowed scientists to greatly expand the size and complexity of the problems they tackle with scientific computing. Sparse polynomials are found in almost every computer application, and zero coefficient polynomials occur frequently in practical settings. In recent years, various algorithms have been designed for the interpolation of a sparse polynomial using a different term basis than the power basis. Prony's classical numerical technique in the 18th century was rediscovered by BenOr and Tiwari and adapted to the computer field nearly 200 years later.

When we look at the situations where sparse polynomials are encountered in practical life, these include problems such as image sharpening and sound waves adjustment. While photo processing and making it close to the real thing have made great progress in
\end{abstract}


recent years, digital images of landscapes such as forests, sunsets and rainbows can be synthesized with unprecedented realism. However, simulating the phenomena associated with the camera lens and sound waves still remains a challenging problem.

In the work to be done, sparse generalized offset curves will be found by approaching the sparse polynomials used in the adjustment of lens blur, aberration and sound waves from a geometric perspective. This sparse generalized offset polynomial curve will be reconstructed using the values of the curve with the Prony algorithm. The relationship between the original sparse polynomial and the sparse generalized offset polynomial curve will be examined. Thus, by using these curves, computer aided geometric design and calculation methods will contribute to fine-tuning the camera lens and sound waves.

Keywords: Sparse Polynomials, Prony Algorithm, Sparse Generalized Offset Polynomial Curve.

\section{Giriş}

Bilgisayar donanımındaki teknolojik gelişmeler, bilim adamlarının bilimsel hesaplama ile ele aldığı sorunların boyutunu ve karmaşıklığını büyük ölçüde genişletmesine izin vermiştir. Bilgisayar programlarının yürütme zamanını tahmin etmek, bilgisayar sistemleri içinde önemli fakat zorlu bir sorundur. Mevcut yöntemler, tahmin ediciler oluşturmak veya önemli özellikleri seçmek için uzmanların program kodunun ayrıntılı analizini yapmalarını gerektirir. Bilgisayar programlarının yürütme zamanını iyileştirmek amacıyla son yıllarda, güç temelinden farklı terim sayısı temelli algoritmalar tasarlanmıştır [1]. Örneğin seyrek polinomlar, basitçe söylemek gerekirse, sıfır katsayıları açıkça saklanmayan bir polinom türüdür. $\mathrm{Bu}$ tür polinom sistemleri her bilimsel disiplinde bulunur ve kritik bir yer tutar. $\mathrm{Bu}$ nedenle doğal olarak bunları işlemek için verimli algoritmalara sahip olmak istenir. Yoğun polinomlar için hızlı algoritmalar artık verimli olmayabileceğinden, seyrek polinomlar için terim temelinin baz alındığı algoritmalar geliştirilmiştir [2]. İnterpolasyon, bazı değerlendirmelerden sonra bilinmeyen bir $\mathrm{f}$ polinomunu öğrenme sürecidir. Seyrek bir polinomun interpolasyonu 18. yüzyılın sonlarında Fransız matematikçi Gaspard de Prony tarafindan bulunmuş olup 1980'lerde Ben-Or ve Tiwari, Blahut ve Zippel ve diğerleri tarafindan yapılan ilerlemeler ile geliştirilmiştir [3].

Paralel eğriler olarak da adlandırılan ofset eğrileri, orijinal eğrilerinden normal vektör boyunca sabit mesafede bulunan noktaların yeri olarak tanımlanır [4]. Bilgisayar destekli geometrik tasarım (CAGD) alanında, ofset eğrileri tolerans analizi, geometrik optik ve robot yol planlama gibi çeşitli pratik uygulamalarda yaygın olarak kullanılmaktadırlar. Günümüzde eğrinin ötelenmesi üzerine yapılan çalışmalar bilgisayar destekli geometrik tasarımın güncel konularından biri olmaya devam etmektedir.

Bu çalışmada amaçlanan, merceklerin odak bulanıklığı ile sapmalarının ve ses dalgalarının ayarlanmasında kullanılan seyrek polinomlara [5] geometrik bir perspektiften yaklaşılarak seyrek genelleştirilmiş ofset eğrilerini bulmaktır. Bu seyrek genelleştirilmiş ofset polinom eğrisi, Prony algoritması ile eğrinin değerleri kullanılarak yeniden elde edilecektir. Orijinal seyrek polinom ile seyrek genelleştirilimiş ofset polinom eğrisi ile arasındaki ilişki incelenecektir. Böylece ofset eğriler kullanılarak kamera mercek ve ses dalgalarının ince ayarlarının yapılması kısmında bilgisayar destekli geometrik tasarım ve hesaplama yöntemleri kullanılarak katkıda bulunulacaktır.

\section{Materyal ve Metot}

\subsection{Prony Algoritması}

Çok değişkenli bir kara kutu polinomunun seyrek interpolasyonu probleminde kara kutu polinomunun hem girdileri hem de çıktıları bazı hatalara sahiptir; köklerinden değerlendirilen kara kutuyu interpolasyon yaparak verimli hale getirmek için çözümler sunulmaktadır [6]. Bir f polinomunun interpolasyonunda polinom sifirdan farklı katsayılara sahip ortogonal polinomların toplamı olarak yazıldıktan sonra orijinal Prony algoritmasında çalıştırılmaktadır. Tek değişkenli bir f polinomunun kara kutudaki keyfi değerlerinden polinomu elde edebiliriz. Ana fikir, ortogonal bazdaki seyrek interpolasyon problemini güç bazında seyrek interpolasyon problemine indirgemek ve ikinci probleme Prony algoritmasını uygulamaktır [7].

\begin{tabular}{|c|c|c|c|c|c|c|c|}
\hline & $u$ & $v$ & $w$ & & & & \\
\hline 1. Chebyshev-1 & 1 & 2 & 0 & $T_{n}(x)$ & $=$ & $V_{n}^{1,2,0,}(x)$ & \\
\hline 2. Chebyshev-2 & 2 & 2 & 0 & $U_{n}(x)$ & $=$ & $V_{n}^{2,2,2,0}(x)$ & \\
\hline 3. Chebyshev-3 & 2 & 2 & -1 & & & & \\
\hline 4. Chebyshev-4 & 2 & 2 & 1 & & & & \\
\hline 5. Dickson-1 & $\frac{1}{2 b}$ & $\frac{1}{6}$ & 0 & $D_{n}\left(x, b^{2}\right)$ & $=$ & $2 b^{n} V_{n}^{\left[\frac{1}{2}, \frac{1}{6}, 0\right]}(x)$ & $=2 b^{n} T_{n}\left(\frac{x}{2 b}\right)$ \\
\hline 6. Dickson-2 & $\frac{1}{b}$ & $\frac{1}{b}$ & 0 & $E_{n}\left(x, b^{2}\right)$ & $=$ & $b^{n} V_{n}^{\left[\frac{1}{b}, \frac{1}{b}, 0\right]}(x)$ & $=b^{n} U_{n}\left(\frac{x}{2 b}\right)$ \\
\hline 7. Dickson- $(k+1)$ & $\frac{1}{(2-k)}$ & & 0 & $n_{n, k}\left(x, b^{2}\right)$ & & k) $b^{n} V_{n}^{[\sqrt{(2)}}$ & \\
\hline
\end{tabular}

Sekil 1: Prony Algortimasinda kullanılan ortogonal polinomlar ve yineleme parametreleri [13].

\subsubsection{Genelleștirilmiş Ofset Ĕgrileri ve bu eğrilerin Prony Algoritması ile Çözülmesi}

Düzlemsel bir parametrik eğri, $\boldsymbol{r}=\boldsymbol{r}(\boldsymbol{t})$ olarak verildiğinde yay uzunluğu parametresine göre S-Frenet çatısında oluşturulan denklemleri $\frac{d e}{d s}=-\kappa \boldsymbol{n}, \frac{d n}{d s}=-\kappa \boldsymbol{e} \quad$ şeklindedir. Burada burada $e, n$ ve $\kappa$ sırasıyla birim teğet vektör, normal vektör ve $r(t)$ eğrisinin eğriliğidir [18]. Serret-Frenet çatısının elemanları kullanılarak düzlemsel $\boldsymbol{r}=\boldsymbol{r}(t)$ eğrisi değişken ofset mesafesi ve ofset yönü ile genelleştirilmiş ofset eğrisi biçiminde yazılabilir: $(t \in[0,1])$ için genelleştirilmiş ofset eğri denklemini $\boldsymbol{r}_{\boldsymbol{o}}(t)=$ $\boldsymbol{r}(t)+d_{1}(t) \boldsymbol{e}+d_{2}(t) \boldsymbol{n}$ olarak verilir ve burada $d_{1}(\mathrm{t})$ ve $d_{2}(t)$, $t$ 'e bağlı birer fonksiyondur [8].

Düzlemsel bir eğri ile düzlemsel eğrinin genelleştirilmiş ofset eğrileri arasındaki ilişkiden yararlanarak ele aldığımız seyrek polinomlar ve seyrek polinomların genelleştirilmiş ofset eğrilerine geometrik bir bakış açısı ile yaklaşılacaktır.

Çalışmada ele alınan seyrek polinomlar ilk olarak polinom eğrisine dönüştürülürken sonrasında Serret-Frenet çatı elemanları kullanılarak seyrek genelleştirilmiş ofset eğrileri bulunacaktır. Genelleştirilmiş ofset eğrinin kara kutu değerlerinden faydalanılarak Prony algoritması ile yeniden üretilmesi sağlanacaktır. Bunlara ek olarak, orjinal seyrek polinom eğrisi ile seyrek genelleştirilmiş ofset eğrileri kıyaslanacaktır. Bu çalışmadaki adımları kısaca özetlersek: 
ADIM 1: Ele alınan seyrek polinomların polinom eğrisine dönüştürülmesi

ADIM 2: Seyrek polinom eğrisinin genelleştirilmiş ofset eğrilerinin bulunması

ADIM 3: Kara kutu değerleri bilinen ofset eğrilerin Prony algoritması kullanılarak değerlerinden yeniden oluşturulması

ADIM 4: Ele alınan seyrek polinomun genelleştirilmiş ofset eğrisi ile Prony algoritmasının ürettiği polinomun karşılaştırılması

\section{Araștırma Sonuçları ve Tartışma}

\subsection{Seyrek Genelleştirilmiş Ofset Polinom Eğrisi ve Prony Algoritması Sonuçları}

Seyrek bir polinom, sıfır katsayıları açıkça saklanmayan polinomlar olup onları işlemek için verimli algoritmalara sahip olmak gerekir. Pratik hayatta birçok uygulamanın içinde var olan seyrek polinomlar özellikle görüntü işleme problemlerinde karşımıza çıkmaktadır. Sentetik görüntülerin gerçek hayattaki görüntülere eşleşmesini maksimum seviyede sağlamak önemli bir problemdir. $\mathrm{Bu}$ çalışmada, optik polinom yaklaşımlarında kullanılan seyrek polinomların kamera lenslerindeki görüntü kalitesini arttırmak için geometrik yaklaşımla genelleştirilmiş ofset eğrilerinden yararlanılmıştır. Bu amaçla, görüntü ve sesin iyileştirilmesinde kullanılan seyrek polinom eğrilerinin seyrek genelleştirilmiş ofset eğrileri oluşturulmuş ve bu ofset eğrisine ait bazı değerler kullanılarak Prony algoritması ile seyrek genelleştirilmiş ofset eğrisi yeniden elde edilmiştir.

\section{Örnek 1:}

$y=x^{3}$ seyrek polinomu için elde edilen seyrek genelleştirilmiş ofset eğrisi ile ilgili sonuçlar Tablo 1 de verilmiştir. Buna ek olarak, $y=x^{3}$ seyrek polinom eğrisi ve seyrek genelleştirilmiş ofset eğrisinin grafikleri Şekil 2 de gösterilmiştir.

Tablo 1: Orjinal seyrek polinom ve Prony Algoritmasının Ürettiği seyrek genelleştirilmiş ofset eğrisi

\begin{tabular}{|c|l|c|}
\hline $\begin{array}{l}\text { Orijinal } \\
\text { Seyrek } \\
\text { Polinom }\end{array}$ & $\begin{array}{l}\text { Seyrek Genelleştirilmiş } \\
\text { Ofset Eğri }\end{array}$ & $\begin{array}{l}\text { Prony } \\
\text { Algoritmasının } \\
\text { Ürettiği Polinom }\end{array}$ \\
\hline$x^{3}$ & $\left(x+1, x^{3}+3 x^{2}, 0\right)$ & $3 x^{3}-3$ \\
\hline
\end{tabular}

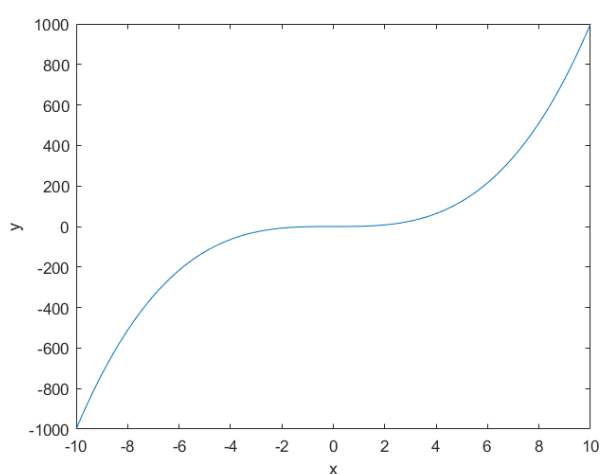

(a)

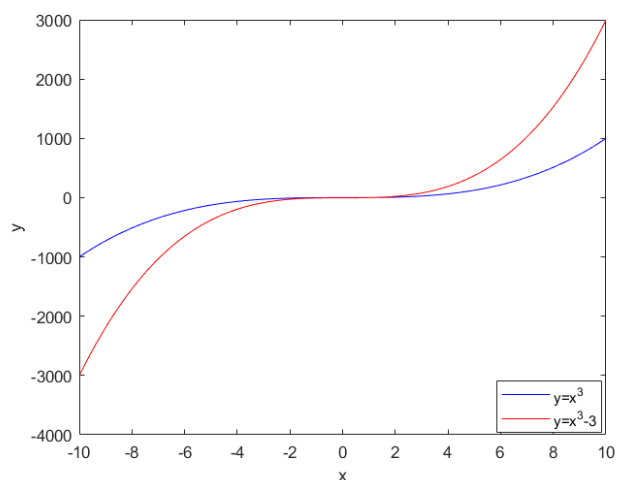

(b)

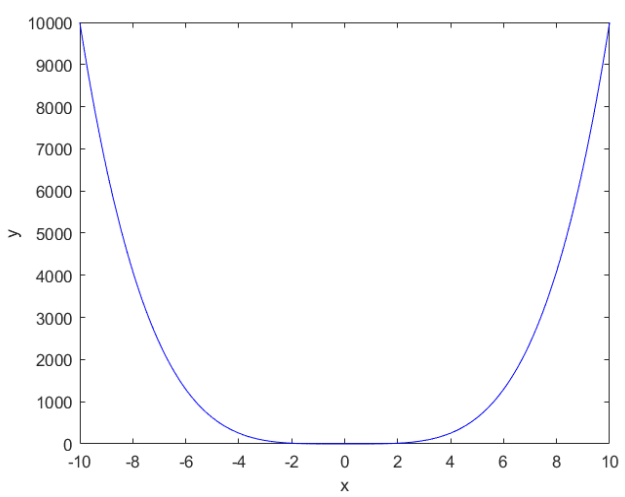

(a)

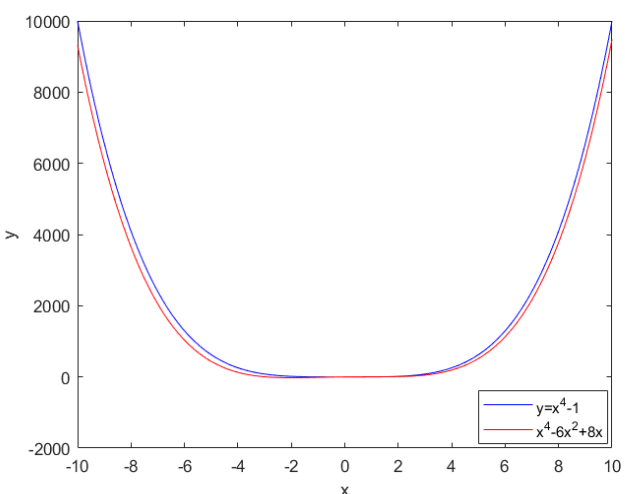

(b)

Şekil 2: Orjinal seyrek polinim eğrisi (a), orjinal seyrek ve seyrek genelleştirilmiş ofset eğrileri (b)

Örnek 2:

$y=x^{4}-1 \quad$ seyrek polinomu için elde edilen seyrek genelleştirilmiş ofset eğrisi ile ilgili sonuçlar Tablo 2 de verilmiştir. Buna ek olarak, $y=x^{4}-1$ seyrek polinom eğrisi ve seyrek genelleştirilmiş ofset eğrisinin grafikleri Şekil 3 de gösterilmiştir.

Tablo 2: Orjinal seyrek polinom ve Prony Algoritmasının Ürettiği seyrek genelleştirilmiş ofset eğrisi

\begin{tabular}{|l|l|l|}
\hline $\begin{array}{l}\text { Orijinal } \\
\text { Seyrek } \\
\text { Polinom }\end{array}$ & $\begin{array}{l}\text { Seyrek Genelleştirilmişs } \\
\text { Ofset Eğri }\end{array}$ & $\begin{array}{l}\text { Prony } \\
\text { Algoritmasının } \\
\text { Ürettiği Polinom }\end{array}$ \\
\hline$x^{4}-1$ & $\left(x+1, x^{4}+4 x^{3}-1,0\right)$ & $x^{4}-6 x^{2}+8 x$ \\
\hline
\end{tabular}


Şekil 3: Orjinal seyrek polinim eğrisi (a), orjinal seyrek ve seyrek genelleştirilmiş ofset eğrileri (b)

Örnek 3:

$y=6 x^{4}$ seyrek polinomu için elde edilen seyrek genelleştirilmiş ofset eğrisi ile ilgili sonuçlar Tablo 3 de verilmiştir. Buna ek olarak, $y=6 x^{4}$ seyrek polinom eğrisi ve seyrek genelleştirilmiş ofset eğrisinin grafikleri Şekil 4 de gösterilmiştir.

Tablo 3: Orjinal seyrek polinom ve Prony Algoritmasının Ürettiği seyrek genelleştirilmiş ofset eğrisi

\begin{tabular}{|c|l|l|}
\hline $\begin{array}{l}\text { Orijinal } \\
\text { Seyrek } \\
\text { Polinom }\end{array}$ & $\begin{array}{l}\text { Seyrek Genelleştirilmişs } \\
\text { Ofset Eğri }\end{array}$ & $\begin{array}{l}\text { Prony } \\
\text { Algoritmasının } \\
\text { Ürettiği Polinom }\end{array}$ \\
\hline $6 x^{4}$ & $\left(x+1,6 x^{4}+24 x^{3}, 0\right)$ & $\begin{array}{l}6 x^{4}-36 x^{2}+48 x \\
-18\end{array}$ \\
\hline
\end{tabular}

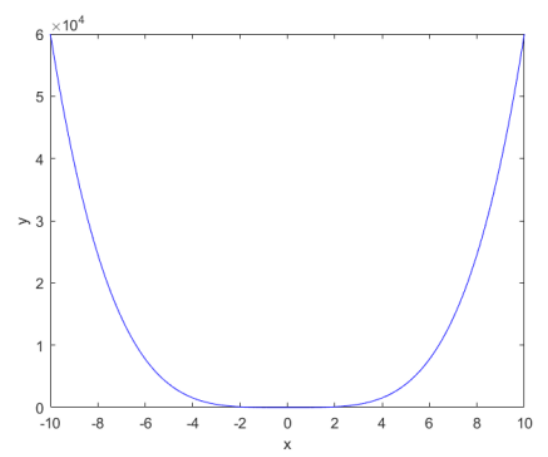

(a)

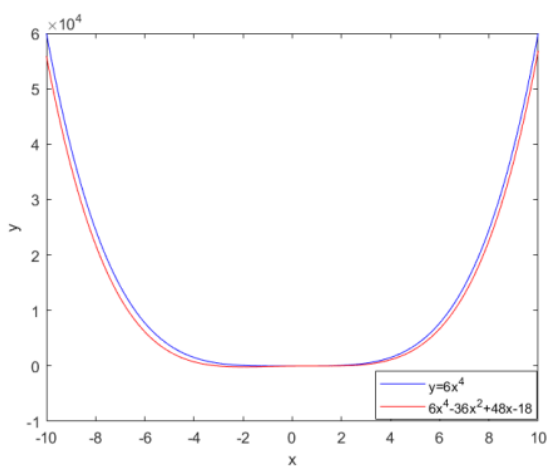

(b)

Şekil 4: Orjinal seyrek polinim eğrisi (a), orjinal seyrek ve seyrek genelleştirilmiş ofset eğrileri (b)

Örneklerden de görüldüğü üzere orjinal seyrek polinom ile seyrek genelleştirilmiş ofset eğrileri kıyaslanarak seyrek genelleştirilmiş ofset eğrisi ile Prony algoritmasının ürettiği polinomların aynı olduğu görülmektedir. Böylece, birden fazla seyrek genelleştirilmiş ofset eğrileri kullanılarak merceklerin odak bulanıklı̆̆ı ile sapmalarının, ve ses dalga ayarlamalarının yapılabileceğini umuyoruz.

\section{Sonuç}

Bu çalışmada, genelleştirilmiş ofset eğrisi kullanılarak seyrek polinomların ofset eğrileri Prony algoritması kullanılarak elde edilmiştir. Genelleştirilmiş seyrek ofset eğrileri kullanılarak kamera mercek ve ses dalgalarının ince ayarlarının yapılmasına bilgisayar destekli geometrik tasarım ve hesaplama perspektiflerinden katkıda bulunulmuştur.

\section{Kaynakça}

1. Asadi M., Brandt A., Moir R.H.C, Maza M.M., (2019). Algorithms and Data Structures for Sparse Polynomial Arithmetic, Mathematics, 7, 441

2. Roche D.S., (2018). What Can (and Can't) we Do with Sparse Polynomials?. ISSAC '18, July 16.

3. Comer M.T., Kaltofen E.L.,Pernet C., (2012). Sparse Polynomial Interpolation and Berlekamp/Massey Algorithms That Correct Outlier Errors in Input Values, ISSAC'12, July 22-25

4. Patrikalakis N.M., (2003). Computational Geometry, $13.472 \mathrm{~J} / 1.128 \mathrm{~J} / 2.158 \mathrm{~J} / 16.940 \mathrm{~J}$

5. Zheng Q., Zheng C., (2017). Adaptive Sparse Polynomial Regression For Camera Lens Simulation, Vis Comput 33:715-724 DOI 10.1007/s00371-017-1402-9

6. Giesbrecht M., Labahna G., Lee W., (2009). SymbolicNumeric Sparse Interpolation of Multivariate Polynomials, Journal of Symbolic Computation 44 (2009) 943-959

7. İmamoğlu E., Kaltofen E.L., (2021). A Note on Sparse Polynomial Interpolation in Dickson Polynomial Basis, ACM Communications in Computer Algebra.

8. Chen X., Lin Q., (2014). Properties of Generalized Offset Curves and Surfaces, Journal of Applied Mathematics, Article ID 124240

9. Kunis S., Peter T., Römer T., Ohe U., (2016). A Multivariate Generalization of Prony's Method, Linear Algebra and its Applications, 490, 31-47

10. Kaltofen E.L., Lee W.S., Yang Z., (2011). Fast Estimates of Hankel Matrix Condition Numbers and Numeric Sparse Interpolation, SNC 2011, June 7-9.

11. Kaltofen E.L., Pernet C., (2021). Sparse Polynomial Interpolation Codes and Their Decoding Beyond Half The Minimal Distance, CCF-1115772, HPAC ANR-11-BS02013.

12. İmamoğlu E., (2021). Sparse Polynomial Interpolation with Bernstein Polynomials, Turk J Math, 45.

13. Imamoglu E., Kaltofen E. L., (2021). A Note on Sparse Polynomial Interpolation in Dickson Polynomial Basis, ACM Communications in Computer Algebra

14. Schrade E., Hanika J., Dachsbacher C., (2016). Sparse HighDegree Polynomials for Wide-Angle Lenses, Eurographics Symposium Volume 35, Number 4

15. Barthélemy Q., Larue A., Mars J., (2015). Color Sparse Representations for Image Processing: Review, Models, and Prospects, HAL Id: hal-01187517

16. Huang L., Jia J., Yu B., Chun B., Maniatis P., Naik M., (2010). Predicting Execution Time of Computer Programs Using Sparse Polynomial Regression, Advances in Neural Information Processing Systems 23

17. Mairal J., Bach F., Ponce J., (2014). Sparse Modeling for Image and Vision Processing, Foundations and Trends in Computer Graphics and Vision, OI: 10.1561/0600000058

18. Bulut V., Çalışkan A., (2015). The Exchange Variations of Offset Curves and Surfaces, Results in Mathematics. 